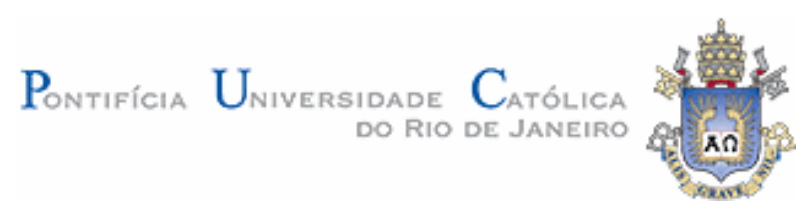

Elaine Alves de Carvalho

\title{
Heurísticas para Identificação de Requisitos de Data \\ Warehouse a partir de Indicadores de Desempenho
}

Dissertação apresentada como requisito parcial para obtenção do título de Mestre pelo Programa de PósGraduação em Informática da PUC-Rio.

Orientador: Rubens Nascimento Melo

Rio de Janeiro, novembro de 2009 


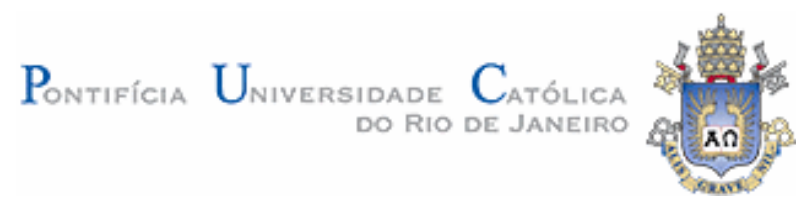

Elaine Alves de Carvalho

\section{Heurísticas para Identificação de Requisitos de Data Warehouse a partir de Indicadores de Desempenho}

Dissertação apresentada como requisito parcial para obtenção do título de Mestre pelo Programa de PósGraduação em Informática da PUC-Rio. Aprovada pela Comissão Examinadora abaixo assinada.

Prof. Rubens Nascimento Melo Orientador Departamento de Informática - PUC-Rio

Prof. Karin Koogan Breitman Departamento de Informática - PUC-Rio

Prof. Flávia Maria Santoro

Unirio

Prof. José Eugenio Leal Coordenador Setorial do Centro Técnico Científico - PUC-Rio 
Todos os direitos reservados. É proibida a reprodução total ou parcial do trabalho sem autorização da universidade, da autora e do orientador.

\section{Elaine Alves de Carvalho}

Graduada em Engenharia de Computação em 2006 pela PUC-Rio. Sua área de interesse acadêmico é Banco de Dados e Engenharia de Requisitos.

Ficha Catalográfica

Carvalho, Elaine Alves

Heurísticas para Elicitação de Requisitos de Data Warehouse a partir de Indicadores de Desempenho / Elaine Alves de Carvalho; orientador: Rubens Nascimento Melo. - Rio de Janeiro: PUC-Rio, Departamento de Informática, 2009.

135f.: il. ; 29,7 cm

Dissertação (mestrado) - Pontifícia Universidade Católica do Rio de Janeiro, Departamento de Informática.

Inclui referências bibliográficas.

1. Informática - Teses. 2. Elicitação de requisitos. 3. Data Warehouse. 4. Indicadores de Desempenho. 5. Processos de Negócio. I. Melo, Rubens Nascimento. II. Pontifícia Universidade Católica do Rio de Janeiro. Departamento de Informática. III. Título. 


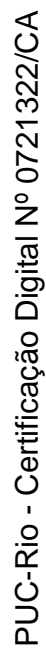

A Deus, meu marido, minha família e amigos, minha sincera gratidão. 


\section{Agradecimentos}

À minha família, mãe, pai (in memoriam) e irmão que, apesar das tarefas exaustivas do dia-a-dia, estiveram sempre presentes, eu agradeço pelas palavras de incentivo e pela paciência. Vocês são e serão sempre a minha base e peças fundamentais na minha vida.

Ao Bruno Santos, meu marido, meu amigo, meu companheiro, meu amor, que me deu forças e me deixou confiante para chegar até aqui. Obrigada pela paciência em me ouvir e ler esse trabalho algumas vezes.

Ao meu orientador Rubens Nascimento Melo que dedicou seu tempo precioso com paciência, atenção e preocupação em meio a tantas outras responsabilidades, mantendo-se sempre presente.

Às professoras Karin Koogan Breitman e Flávia Maria Santoro por aceitarem participar dessa banca de mestrado. Estou certa de que suas contribuições permitirão o desenvolvimento futuro dessa pesquisa.

Aos amigos Carlos Portela, Tarcísio, Lúcia, Edmar, Tatiana e Jairo pelo auxílio, orientação e suporte.

Finalmente, aos amigos do Departamento de Informática e a todas as pessoas que, de alguma forma, contribuíram para que eu conseguisse tornar realidade esse trabalho tão almejado. 


\section{Resumo}

Carvalho, Elaine Alves; Melo, Rubens Nascimento. Heurísticas para Identificação de Requisitos de Data Warehouse a partir de Indicadores de Desempenho. Rio de Janeiro, 2009. 135p. Dissertação de Mestrado Departamento de Informática, Pontifícia Universidade Católica do Rio de Janeiro.

As organizações se deparam com uma necessidade cada vez maior de mudar e evoluir, mas para isso elas precisam tomar as decisões corretas. Para essa tomada de decisão, as empresas estão adotando os recursos disponibilizados pela Tecnologia da Informação (TI) como parte fundamental para apoiar suas decisões. Um componente de $\mathrm{Tl}$ essencial para aprimorar o processo de tomada de decisão é o data warehouse. Para cumprir bem o seu papel, o data warehouse deve ser bem definido. Embora existam diversas abordagens que buscam melhorar a tarefa de identificação dos requisitos para data warehouses, poucas exploram as contribuições da Engenharia de Processos de Negócios (EPN) no processo de definição dos requisitos. Esta dissertação estuda um meio de aprimorar a tarefa de elicitação de requisitos para data warehouses, utilizando indicadores de desempenho aliados aos processos de negócio. Para isso é sugerido um conjunto de heurísticas que visam, a partir dos indicadores de desempenho, orientar a descoberta dos requisitos de data warehouse. A aplicação das heurísticas propostas é feita em um caso, facilitando a compreensão da abordagem sugerida nesse trabalho.

\section{Palavras-chave}

Elicitação de requisitos; data warehouse; indicadores de desempenho; processos de negócio. 


\section{Abstract}

Carvalho, Elaine Alves; Melo, Rubens Nascimento (Advisor). Heuristics for Data Warehouse Requirements Elicitation Using Performance Indicators. Rio de Janeiro, 2009. 135p. MSc. Dissertation - Departamento de Informática, Pontifícia Universidade Católica do Rio de Janeiro.

Organizations need to change and evolve, but for that it is necessary to make the right decisions. For this decision, companies are using Information Technology (IT) as a fundamental part to support their decisions. An essential IT component to improve the process of decision making is the data warehouse. In order to fulfill its role well, the data warehouse must be well defined. There are various approaches that try to improve the task of identifying data warehouses requirements, but few explore the contributions of Business Processes Engineering (BPE) in the process of requirements gathering. This dissertation studies how to improve data warehouses requirements elicitation using performance indicators allied to business processes. For this it is suggested a set of heuristics designed to guide performance measures identification and data warehouse requirements discovery. The heuristics are applied in a case to facilitate understanding of suggested approach in this work.

\section{Keywords}

Requirements elicitation; data warehouse; performance indicators; business processes. 


\section{Sumário}

1 Introdução 17

$\begin{array}{ll}1.1 \text { Motivação } & 17\end{array}$

1.2 Questões da Pesquisa 20

$\begin{array}{ll}1.3 \text { Justificativa } & 23\end{array}$

$\begin{array}{ll}1.4 \text { Objetivos } & 27\end{array}$

1.5 Delimitações 28

$\begin{array}{ll}1.6 \text { Estrutura da Dissertação } & 29\end{array}$

2 Processos de Negócios e Indicadores de Desempenho 31

2.1 Definindo a Engenharia de Processos de Negócios (EPN) 31

2.2 Processos de Negócios 33

2.2.1 Definindo o conceito de processo de negócio 33

2.2.2 Conceitos da modelagem de processo de negócio 36

2.2.3 Aplicações da Engenharia de Processos de Negócios 37

2.2.4 Modelagem de processos de negócios 40

2.2.5 Princípios da modelagem de processos 42

2.2.6 Níveis de agregação dos processos 43

2.2.7 Alguns métodos de modelagem de processos 44

2.2.7.1 ARIS - Arquitetura de Sistema de Informação Integrados 44

2.2.7.2 CIMOSA - Arquitetura Aberta de Sistemas CIM 47

2.2.7.3 IDEF - Métodos Integrados de Definição 47

2.2.7.4 BPMN - Notação de Modelagem de Processos de Negócio 49

2.2.8 Ferramentas de Modelagem 52

2.3 Indicadores de Desempenho 52

2.4 Considerações Finais $\quad 54$

3 Data Warehouse $\quad 56$

3.1 Sistemas Data Warehouse 56

3.2 Data Warehousing $\quad 57$

3.3 Requisitos para Data Warehouses $\quad 59$

3.3.1 Definindo Requisitos 63

3.4 O Problema da Elicitação de Requisitos para Data Warehouse 68 
4 Heurísticas para Definição de Requisitos para Data Warehouses

4.1 Definindo Heurísticas

4.2 Visão Geral

4.3 Heurísticas Propostas $\quad 77$

4.3.1 Regras Gerais (R) 80

4.3.2 Heurísticas de Identificação dos Indicadores (HII) 81

4.3.3 Heurísticas de Identificação de Processos de Negócio (HIP) 82

4.3.4 Heurísticas de Identificação de Associações (HIA) 83

4.3.5 Heurísticas de Identificação das Fontes de Informação (HIF) 84

4.4 Considerações Finais $\quad 85$

5 Aplicação das Heurísticas - Caso de uma Empresa de Manutenção 86

5.1 Apresentação do Caso 86

5.2 Aplicação das Heurísticas $\quad 88$

5.2.1 Entendimento dos objetivos estratégicos $\quad 89$

$\begin{array}{ll}\text { 5.2.2 Desdobramento dos objetivos estratégicos } & 91\end{array}$

5.2.3 Desdobramento dos objetivos operacionais 93

$\begin{array}{ll}5.2 .4 \text { Identificação das fontes de informação } & 97\end{array}$

5.2.5 Identificação dos indicadores gerenciais e estratégicos 99

5.3 Considerações Finais 101

6 Trabalhos Relacionados 102

6.1 Abordagens Orientadas para Desenvolvimento de Data Warehouse 102

6.2 Abordagens com Foco no Processo de Elicitação de Requisitos de Data

$\begin{array}{ll}\text { Warehouses } & 103\end{array}$

6.3 Abordagens Baseadas em Processos de Negócio 105

$\begin{array}{ll}6.4 \text { Considerações Finais } & 106\end{array}$

7 Conclusão e Trabalhos Futuros 107

$\begin{array}{ll}7.1 \text { Retrospectiva do Trabalho Apresentado } & 107\end{array}$

$\begin{array}{ll}7.2 \text { Trabalhos Futuros } & 109\end{array}$

8 Referências 112

9 Anexo 1 - Taxonomia dos Requisitos Não Funcionais 124 
10 Anexo 2 - Modelos de Registro para Aplicação das Heurísticas

11 Anexo 3 - Resultados da Aplicação das Heurísticas 


\section{Lista de figuras}

Figura 1 - Localização temática do enfoque da solução 23

$\begin{array}{ll}\text { Figura } 2 \text { - Aplicações de processos } & 38\end{array}$

Figura 3 - Visão funcional versus visão processual. 42

Figura 4 - ARIS House: da estratégia ao sistema de informação orientado por processos 46

Figura 5 - Métodos IDEF: partes da ferramenta para engenharia de sistemas 48

Figura 6 - Tipologia de ferramentas de auxilio à modelagem de processos 52

Figura 7 - O processo de DWing. 58

Figura 8 - Processo da Engenharia de Requisitos 60

Figura 9 - Hierarquia de requisitos $\quad 66$

Figura 10 - Visão top-down de alinhamento dos objetivos e bottom-up de indicadores para acompanhamento da estratégia nos diversos níveis organizacionais 73

Figura 11 - Níveis hierárquicos dos indicadores de desempenho. $\quad 74$

Figura 12 - Indicadores de resultado e de tendência. 75

Figura 13 - Relação entre indicadores e detalhamentos dos processos de negócio 76

Figura 14 - Cadeia de Valor de nível mais agregado da ESM 87

Figura 15 - Objetivos estratégicos da ESM 88

Figura 16 - Diagrama de Objetivos Estratégicos da ESM 89

Figura 17 - Objetivos gerenciais derivados de um objetivo estratégico 91

Figura 18 - Cadeia de Valor de segundo nível 92

Figura 19 - Desdobramento dos objetivos gerenciais em operacionais e suas $\begin{array}{ll}\text { associações } & 94\end{array}$

Figura 20 - Relação dos indicadores operacionais propostos para o fluxo de atividades 96

Figura 21 - Visualização da complexidade da atividade associada a um indicador operacional $\quad 98$

Figura 22 - Estágios de detalhamento 105

Figura 23 - Tipos de requisitos não funcionais $\quad 124$

Figura 24 - Modelo de registro da associação entre níveis de detalhamento dos processos e objetivos 125

Figura 25 - Modelo de registro dos indicadores de desempenho com 


$$
\begin{aligned}
& \begin{array}{l}
\text { associação aos processos de negócio nos diversos níveis de } \\
\text { detalhamento }
\end{array} \\
& 126
\end{aligned}
$$

Figura 26 - Modelo de registro dos indicadores de desempenho com $\begin{array}{ll}\text { associação às atividades dos processos de negócio } & 127\end{array}$ 


\section{Lista de tabelas}

Tabela 1 - Resumo das Características e Representação dos Elementos BPMN

Tabela 2 - Regras Gerais

Tabela 3 - Heurísticas de Identificação de Indicadores (HII)

Tabela 4 - Heurísticas de Identificação de Processos de Negócio (HIP)

Tabela 5 - Heurísticas de Identificação de Associações (HIA)

Tabela 6 - Heurísticas de Identificação de Fontes de Informação (HIF)

Tabela 7 - Exemplo de Indicador de Desempenho Obtido pela Aplicação das Heurísticas

Tabela 8 - Exemplo de Requisito Obtido para o DW

Tabela 9 - Modelo de Registro de Requisitos de Informação do DW

Tabela 10 - Lista de Indicadores Estratégicos da ESM 129

Tabela 11 - Exemplo de Indicadores de Desempenho da ESM 130

Tabela 12 - Exemplo de Requisitos do DW da ESM 


\section{Lista de siglas e abreviaturas}

\begin{tabular}{|c|c|}
\hline ARIS & $\begin{array}{l}\text { Architecture of Integrated Information Systems (Arquitetura de } \\
\text { Sistemas Integrados de Informação) }\end{array}$ \\
\hline BPMN & $\begin{array}{l}\text { Business Process Modeling Notation (Notação de Modelagem de } \\
\text { Processos de Negócio) }\end{array}$ \\
\hline BPR & $\begin{array}{l}\text { Business Process Reengineering (Reengenharia de Processos de } \\
\text { Negócios) }\end{array}$ \\
\hline BRG & Business Rules Group \\
\hline BROCOM & $\begin{array}{l}\text { Business Rule Oriented Conceptual Modeling (Modelagem } \\
\text { Conceitual Orientada a Regra de Negócio) }\end{array}$ \\
\hline CEO & Chief Executive Officer (Executivo-Chefe) \\
\hline CIM & $\begin{array}{l}\text { Computer Integrated Manufacturing (Manufatura Integrada por } \\
\text { Computador) }\end{array}$ \\
\hline CIM & $\begin{array}{l}\text { Computer Independent Model (Modelo Independente } \\
\text { Computador) }\end{array}$ \\
\hline CIMOSA & $\begin{array}{l}\text { Computer Integrated Manufacturing Open System Architecture } \\
\text { (Arquitetura Aberta de Sistemas CIM) }\end{array}$ \\
\hline $\mathrm{ClO}$ & Chief Information Officer (Executivo-Chefe de TI) \\
\hline CSP & $\begin{array}{l}\text { Communicating Sequential Process (Processo Sequencial de } \\
\text { Comunicação) }\end{array}$ \\
\hline DER & $\begin{array}{l}\text { Extended Entity Relationship Model (Diagrama de Entidades- } \\
\text { Relacionamento) }\end{array}$ \\
\hline DFD & Diagrama de Fluxo de Dados \\
\hline DO & Diagrama de Objetivos \\
\hline DSS & Decision Support System (Sistema de Apoio à Decisão) \\
\hline DW & Data Warehouse (Armazém de Dados) \\
\hline DWing & Data Warehousing (Processo de construção de DW) \\
\hline ECA & Event-Condition-Action (Evento-Condição-Acão) \\
\hline EIS & Enterprise Information System (Sistema de Informação Executiva) \\
\hline EPC & $\begin{array}{l}\text { Event-driven Process Chain (Cadeia de Processos Orientada por } \\
\text { Eventos) }\end{array}$ \\
\hline EPN & Engenharia de Processos de Negócios \\
\hline ER & Engenharia de $\mathrm{R}$ \\
\hline ERM & p Model (Modelo Entidade Relacionamento) \\
\hline
\end{tabular}




\begin{tabular}{|c|c|}
\hline ERP & $\begin{array}{l}\text { Enterprise Resource Planning (Sistemas de Planejamento de } \\
\text { Recursos do Negócio) }\end{array}$ \\
\hline ES & Engenharia de Software \\
\hline ETL & $\begin{array}{l}\text { Extraction, Transformation and Load (Extracao, Transformação e } \\
\text { Carga) }\end{array}$ \\
\hline FAD & Function Allocation Diagram (Diagrama de Função) \\
\hline FT & Function Tree (Árvore de Funções) \\
\hline IDEF & Integration Definition Methods (Métodos Integrados de Definição) \\
\hline IS & Information System (Sistema de Informação) \\
\hline ISO & International Standard Organization \\
\hline IT & Information Technology (Tecnologia da Informação) \\
\hline KAOS & Knowledge Acquisition in autOmated Specification \\
\hline KSD & Knowledge Structure Diagram (Estrutura de Conhecimento) \\
\hline MDA & Model-Driven Architecture (Arquitetura Dirigida por Modelo) \\
\hline MDS & Metodologia de Desenvolvimento de Sistemas (Software) \\
\hline OCL & Object Constraint Language (Linguagem de Restrição de Objeto) \\
\hline OMT & Object Modeling Technique (Técnica de Modelagem a Objeto) \\
\hline OMG & Object Management Group \\
\hline $\mathrm{OO}$ & Object Oriented (Orientação a Objeto) \\
\hline OOA & Object Oriented Analysis (Análise Orientada a Objeto) \\
\hline ORG & Organizational Chart (Organograma) \\
\hline OSTN & $\begin{array}{l}\text { Object State Transition Network (Rede de Transição de Estado de } \\
\text { Objeto) }\end{array}$ \\
\hline PIM & $\begin{array}{l}\text { Platform Independent Model (Sistema de Plataforma } \\
\text { Independente) }\end{array}$ \\
\hline PSM & Platform Specific Model (Sistema de Plataforma Específica) \\
\hline RAD & Role Activity Diagrams (Diagrama de Papéis e Atividades) \\
\hline RFI & Request For Information (Requisição de Informação) \\
\hline RFP & Request For Proposal (Requisição de Proposta) \\
\hline RUP & Rational Unified Process (Processo Unificado da Rational) \\
\hline SADT & $\begin{array}{l}\text { Structured Analysis and Design Technique (Técnica de Projeto e } \\
\text { Análise Estruturada) }\end{array}$ \\
\hline SD & Strategic Dependence (Dependência Estratégica) \\
\hline SERM & $\begin{array}{l}\text { Structured Entity Relationship Model (Modelo Entidade } \\
\text { Relacionamento Estruturado) }\end{array}$ \\
\hline$c$ & Sistema de Informação \\
\hline
\end{tabular}


SR Strategic Rationale (Estratégia Racional)

TI Tecnologia da Informação

Udl Universo de Informações

UML Unified Modeling Language (Linguagem Unificada de Modelagem)

WFM Workflow Management (Gerenciamento do Fluxo de Trabalho)

VAC Value-Added Chain Diagram (Diagrama de Cadeia de Valor Agregado) 